\title{
Analysis of the Factors Affecting the Accuracy of Numerical Simulation of Polymer Flooding
}

\author{
Baotao Zhou \\ No.5 Oil Production Plant of Daqing Oilfield Company, Daqing, 163315, China \\ zhoubaotao123@163.com
}

Keywords: Polymer Flooding, Numerical Simulation, Sensitivity Analysis

\begin{abstract}
Numerical simulation is of great importance as implementation guides of polymer flooding projects. The history matching process is one of the main tasks of numerical simulation. However, there are still significant difficulties remaining in the history matching process of polymer flooding simulation due to uncertainties of some related parameters in polymer flooding. This article selects a block as a research block, the main factors affecting the fitting effect are clearly defined by comparing the simulation results of the simulation parameters under different factors in the block. It is of great significance to the guidance and adjustment of block tracking. It has reference significance for other block polymer flooding history matching.
\end{abstract}

\section{Introduction}

With the full implementation of polymer flooding technology in Daqing oilfield, the understanding of polymer flooding theory has deepened. A large number of new technologies for improving polymer flooding efficiency have been applied to polymer flooding production practice. Compared with the conventional water flooding, the new technology and new method of polymer flooding have higher technical, higher cost and greater risk. It is very necessary to use the numerical simulation method to guide them scientifically. Through numerical simulation to track the fitting and prediction results, we can scientifically track and adjust the remaining oil distribution in polymer flooding process. This raises higher requirements for the accuracy of polymer flooding numerical simulation.

The numerical simulation expert of Daqing oilfield put forward the concept of polymer solution reservoir working parameter that affects the accuracy of numerical simulation and prediction. The so-called polymer solution reservoir working parameter is the physical and chemical index of polymer solution under the condition of oil reservoir. It includes four contents: viscosity concentration relationship curve, rheology behavior, non porous volume and residual resistance coefficient. A block is the first polymer flooding industrial block in our plant, through the blank water drive, water cut, water cut and water cut up period, water flooding has been fully entered, It has experienced a more complete development process of polymer flooding. Through the historical fitting of polymer flooding stage in the polymer flooding industrial area of the thirteen apricot area, the influence degree of different reservoir working parameters on the accuracy of polymer flooding numerical simulation is clarified. The effects of four polymer solution reservoir parameters on the fitting results are introduced below.

\section{Viscosity of Polymer Solution}

The high viscosity of polymer solution can improve the oil-water mobility ratio, inhibit the sudden development of injected water, and enlarge the macroscopic wave volume. The experimental results show that the viscosity of polymer solution is a function of concentration and salt content of polymer solution at zero shear rate. The following function is expressed as:

$$
\mu_{\mathrm{p}}^{0}=\mu_{\mathrm{w}}\left(1+\left(\mathrm{A}_{\mathrm{p} 1} \times \mathrm{C}_{\mathrm{p}}+\mathrm{A}_{\mathrm{p} 2} \times \mathrm{C}_{\mathrm{p}}^{2}+\mathrm{A}_{\mathrm{p} 3} \times \mathrm{C}_{\mathrm{p}}^{3}\right) \times \mathrm{C}_{\mathrm{SEP}}\right)
$$


In the type: $\mathrm{C}_{\mathrm{p}}$ is the concentration of the polymer in a solution; $\mu_{\mathrm{w}}$ is the aqueous viscosity.

The relationship between viscosity $\mu_{\mathrm{p}}{ }^{0}$ and polymer concentration $C_{p}$ is expressed by an incomplete three degree polynomial. The coefficients AP1, AP2 and AP3 of the polynomial are constants determined by experimental data.

The relation between viscosity and salt content is the form of power function, in the relation: $\mathrm{C}_{\mathrm{SEP}}$ is the effective salt concentration of polymer; $\mathrm{S}_{\mathrm{p}}$ is the parameters determined by the experiment.

Contrast with three sets of data: (see table 1).

Table 1 Parameter values for different thick viscosity relations

\begin{tabular}{|c|c|c|c|c|c|c|}
\hline \multirow{2}{*}{ Parameter } & \multicolumn{3}{|c|}{ Polymer 1 } & \multicolumn{3}{c|}{ Polymer 2 } \\
\cline { 2 - 8 } & $\mathrm{A}_{\mathrm{p} 1}$ & $\mathrm{~A}_{\mathrm{p} 2}$ & $\mathrm{~A}_{\mathrm{p} 3}$ & $\mathrm{~A}_{\mathrm{p} 1}$ & $\mathrm{~A}_{\mathrm{p} 2}$ & $\mathrm{~A}_{\mathrm{p} 3}$ \\
\hline Relationship 1 between viscosity and concentration & 1 & 135 & 300 & 1 & 50 & 500 \\
\hline Relationship 2 between viscosity and concentration & 2 & 270 & 300 & 2 & 100 & 1000 \\
\hline Relationship 3 between viscosity and concentration & 0.5 & 67.5 & 150 & 0.5 & 25 & 250 \\
\hline
\end{tabular}

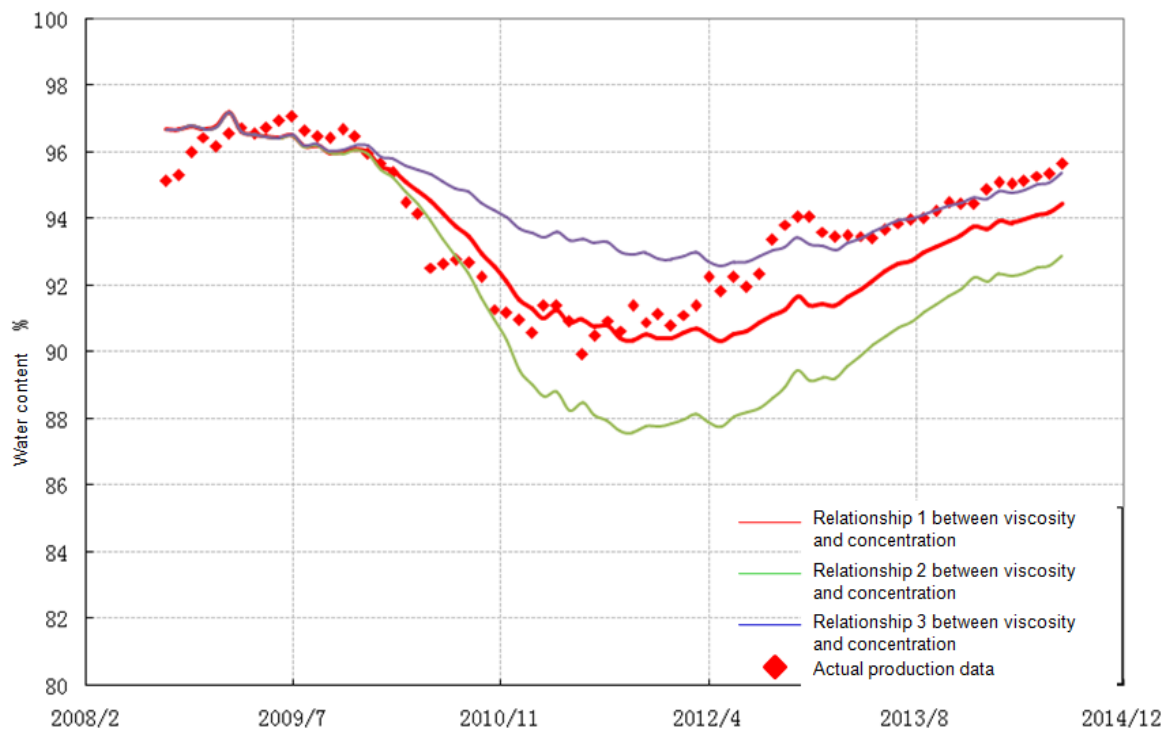

Fig .1. Comparison of fitting effect of different thick viscosity relationship

Through comparison (see Figure 1), it can be seen that different thick viscosity relations have great influence on the results of fitting or prediction, and the concentrated viscosity curve mainly affects the decrease of water cut which is fitted or predicted, which has little influence on the time of water cut.

\section{Rheological Characteristics of Polymer Solution}

The polymer solution has rheological properties, and its viscosity depends on the shear rate .This dependence is expressed by the Meter equation. The viscosity of polymer solution $\mu_{\mathrm{p}}$ is related to the shear rate:

$$
\mu_{\mathrm{p}}=\mu_{\mathrm{w}}+\frac{\mu_{\mathrm{p}}^{0}-\mu_{\mathrm{w}}}{1+\left(\gamma^{\mathrm{ref}}\right)^{\mathrm{p} \alpha-1}}
$$

$\gamma_{\text {ref }}$ is the reference shear rate; $\mathrm{p}_{\alpha}$ is the coefficient determined by experimental data; $\mu_{\mathrm{p}}$ is called the apparent viscosity of polymer solution flowing in porous medium; $\gamma$ is the equivalent shear rate of fluid in porous media.

The equivalent shear rate of water in porous media $\gamma$ is expressed by Blake-Kozeny equation.

$$
\gamma=\frac{\gamma_{\mathrm{c}} \times\left|\mu_{\mathrm{w}}\right|}{\sqrt{\mathrm{k} \times \mathrm{k}_{\mathrm{rw}} \times \emptyset \times \mathrm{S}_{\mathrm{w}}}}
$$


$\gamma_{c}$ is the shear rate coefficient is related to the non ideal effect (for example, the slip phenomenon of capillary wall in porous media); $\mathrm{k}_{\mathrm{rw}}$-Relative permeability of water phase.

Contrast with two sets of data(see table 2):

Table 2 Parameters used for different shear rates

\begin{tabular}{|c|c|c|}
\hline Parameter & $\gamma_{c}$ & $p_{a}$ \\
\hline Shear rates 1 & 30.25 & 1.572 \\
\hline Shear rates 2 & 10 & 0.572 \\
\hline
\end{tabular}

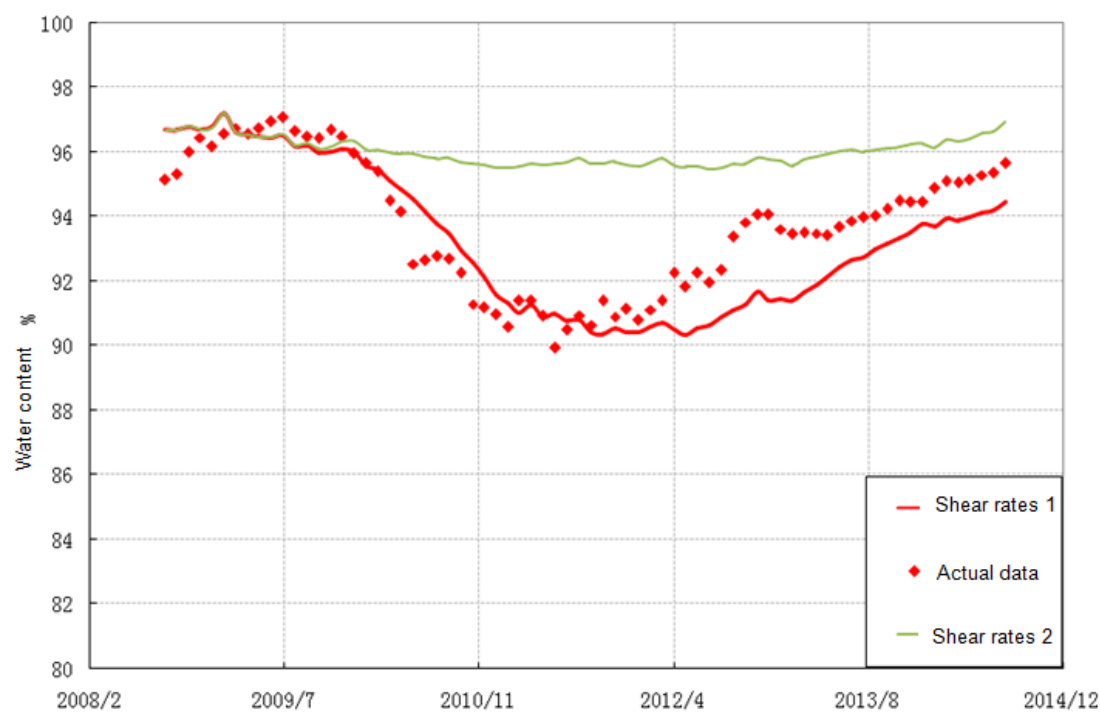

Fig .2. Comparison of fitting effects of different shear rates

By contrast (as in Figure 2), it can be seen that the shear rate has great influence on the results of fitting or prediction, and the shear rate mainly affects the decrease of water cut, which has little effect on the time of water cut.

\section{Coefficient Of Permeability Decline in Water Phase}

When polymer solution flows in porous media, the absorption and capture of polymer in porous medium will cause the decrease of mobility and increase of flow resistance. This phenomenon is described by the permeability descent coefficient Rk.

$$
\begin{aligned}
& \mathrm{R}_{\mathrm{k}}=1+\frac{\left(\mathrm{R}_{\mathrm{KMAX}}-1\right) \times \mathrm{b}_{\mathrm{rk}} \times \mathrm{C}_{\mathrm{p}}}{1+\mathrm{b}_{\mathrm{rk}} \times \mathrm{C}_{\mathrm{p}}} \\
& \mathrm{R}_{\mathrm{KMAX}}=\left[1-\left(\mathrm{C}_{\mathrm{rk} \times} \mu_{\mathrm{peff}} 1 / 3 /\left(\frac{\sqrt{\mathrm{k}_{\mathrm{x}} \times \mathrm{k}_{\mathrm{y}}}}{\emptyset \times \mathrm{S}_{\mathrm{w}}}\right)^{1 / 2}\right)\right]^{-4} \\
& \mu_{\mathrm{peff}}=\lim _{\mathrm{C}_{\mathrm{p}} \rightarrow 0} \frac{\mu_{0}-\mu_{\mathrm{w}}}{\mu_{\mathrm{w}} \times \mathrm{C}_{\mathrm{p}}}=\mathrm{A}_{\mathrm{p} 1} \times \mathrm{C}_{\mathrm{sep}}
\end{aligned}
$$

In the type: $R_{k}$ is the permeability descent coefficient; $C_{p}$ is the Mass concentration of polymer flooding in solution; $\Phi$ is the Porosity; $S_{w}$ is the water saturation; $b_{r k}$ is the input parameters; $c_{r k}$ is the experimental data determination; $\mathrm{R}_{\mathrm{kmax}}$ is the maximum permeability decline coefficient; $\mu_{\mathrm{peff}}$ is the Intrinsic viscosity of polymer solution.

Compare with three sets of data(see table 3):

Table 3 Parameters used for different permeability decline coefficients

\begin{tabular}{|c|c|c|c|}
\hline Parameter & $\begin{array}{c}\text { Permeability descent coefficient } \\
1\end{array}$ & $\begin{array}{c}\text { Permeability descent } \\
\text { coefficient 2 }\end{array}$ & $\begin{array}{c}\text { Permeability descent } \\
\text { coefficient 3 }\end{array}$ \\
\hline $\mathrm{b}_{\mathrm{rk}}$ & 20 & 10 & 30 \\
\hline
\end{tabular}


Through comparison (see figure3),it can be seen that the coefficient of permeability decrease has great influence on the results of fitting or prediction, and it mainly affects the decrease of water cut, which has little effect on the time of water cut.

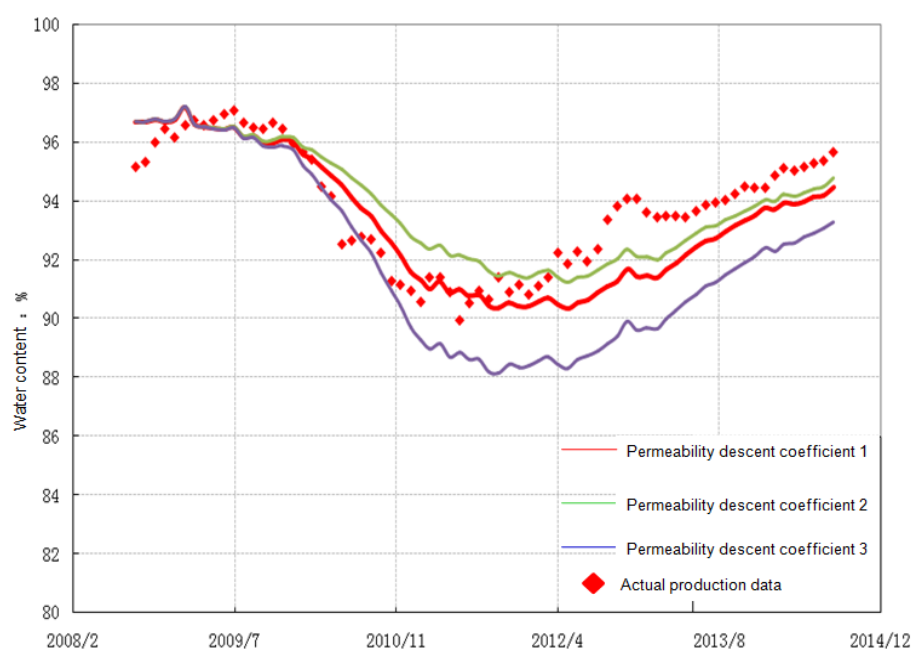

Fig. 3. Comparison of the fitting effect of different permeability decline coefficients

\section{Inaccessible Pore Volume}

It is found that the flow of polymer is faster than that of a tracer in porous media. This can be explained by the small pore volume that the polymer can flow through, which is determined by the polymer structure of the polymer. The volume of pores that the polymer can't enter is called inaccessible pore volume. In the model, it is expressed as:

$$
\mathrm{IPV}=\frac{\emptyset-\emptyset_{\mathrm{p}}}{\emptyset}
$$

In the type: IPV is the inaccessible pore volume fraction of polymer solution; $\varphi$ is the porosity measured for brine; $\varphi_{\mathrm{p}}$ is the porosity measured for polymer solutions.

Contrast with two sets of data (see table 4):

Table 4 Parameter values for different void volume

\begin{tabular}{|c|c|c|c|}
\hline Parameter & Inaccessible pore volume 1 & Inaccessible pore volume 2 & Inaccessible pore volume 3 \\
\hline IPV & 0.22 & 0.17 & 0.12 \\
\hline
\end{tabular}

Through comparison (see figure 4), it can be seen that the inaccessible pore volume has less influence on the results of fitting or prediction, and the inaccessible pore volume affect the time of water cut, which has little effect on the decrease of water cut.

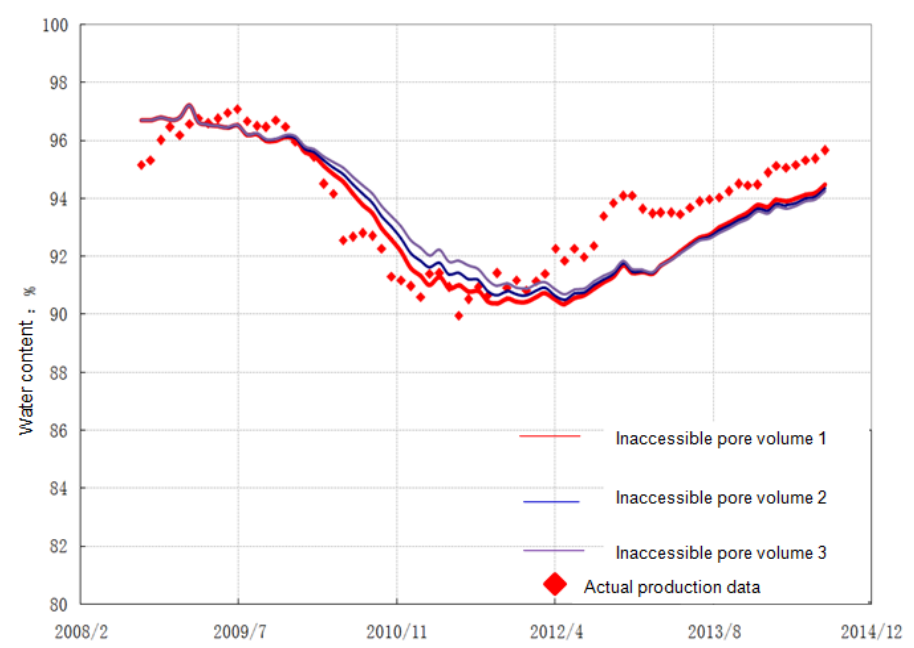

Fig. 4. Comparison of fitting effect of different inaccessible pore volume 


\section{Conclusion}

Different polymer reservoir parameters have different effects on polymer flooding fitting or prediction. The influence of the viscosity of polymer solution, the rheological characteristics of the polymer solution and the coefficient of water permeability decrease on the fitting or prediction of polymer flooding is mainly reflected in the decrease of water cut, which has little influence on the time of water cut. The influence of pore volume on the fitting or prediction of polymer flooding is mainly reflected in the effect of water cut down time, and on the decrease of water cut.

\section{References}

[1] Wang Qimin, Liao Guangzhi, Niu Jingang.Practice and understanding of polymer flooding technology [J]. Daqing petroleum geology and development, 1999,18(4):1-5.

[2] Zhang Zhongxun,Shen Quanfu,Chen Wenruo,et a1.Reservoir multidisciplinary research and application of polymer flooding[M].Beijing: Petroleum Industry Press, 2014:154-165.

[3] Wang Xinhai, Han Dakuang, Guo Shangping. Polymer flooding mechanism and application [J]. Journal of petroleum science, 1994,25(1):83-91. 